\title{
QUANTIZATION OF CURVATURE OF HARMONIC TWO-SPHERES IN GRASSMANN MANIFOLDS
}

\author{
YUNBO ZHENG
}

\begin{abstract}
Various pinching theorems for curvature of minimal two-spheres in Grassmann manifolds have been proved. In particular, we show that when the curvature is large, then the minimal map from $S^{2}$ into $G(m, N)$ must be either holomorphic or antiholomorphic. Also, minimal two-spheres of curvature $\kappa \geq 2$ in $G(2,4)$ have been classified.
\end{abstract}

\section{INTRODUCTION}

It is known that starting from a harmonic map $f$ from a Riemann surface into a complex Grassmann manifold, one can define sequences of harmonic maps by using the $\partial$-transform and the $\bar{\partial}$-transform. These sequences are called harmonic sequences [CW]. When $f$ is holomorphic, the sequence generated by $f$ via the $\partial$-transform is called a pseudoholomorphic sequence, and the corresponding maps are called pseudoholomorphic maps.

In 1980 A. M. Din and W. J. Zakrzewski [DZ], and then in 1983 J. Eells and J. C. Wood [EC], showed that all harmonic maps from $S^{2}$ to the complex projective space $C P^{n}$ are, in fact, pseudoholomorphic. Recently, F. Burstall, J. Rawnsley and S. Salamon announced in [BRS] that all stable harmonic maps from $S^{2}$ into irreducible Hermitian symmetric spaces are either holomorphic or antiholomorphic. It is also known that when the target space is a general complex Grassmann manifold, holomorphic curves do not generate all harmonic maps [CW], [Ra].

On the other hand, M. Rigoli showed in [R] that for a linearly full nonsingular holomorphic curve from $S^{2}$ into $C P^{n}$, if its curvature $\kappa \geq 4 / n$, then $\kappa=4 / n$ is constant. Then J. Bolton et al. [BJRW] generalized this result to pseudoholomorphic maps, and showed that for a linearly full isometric pseudoholomorphic map of position $r$ from $S^{2}$ into $C P^{n}$, if its curvature $\kappa \geq 4 /(2 r(n-r)+n)$, then $\kappa=4 /(2 r(n-r)+n)$ is constant.

In this paper, we prove various pinching theorems of curvature for harmonic maps and show that for any nonsingular harmonic map from $S^{2}$ into a complex Grassmann manifold, if the curvature of its induced metric is large, then

Received by the editors January 18, 1988 and, in revised form, April 11, 1988.

1980 Mathematics Subject Classification (1985 Revision). Primary 53A10, 53C55.

Key words and phrases. Harmonic maps, pseudoholomorphic, pinching. 
it is actually a holomorphic or antiholomorphic curve of constant curvature. Precisely, we show that for any harmonic isometric immersion from $S^{2}$ to $G(m, N)$, if it is not antiholomorphic (or not holomorphic), and if its curvature $\kappa \geq 4 / \operatorname{rank}(\partial f)$ (or $\kappa \geq 4 / \operatorname{rank}(\bar{\partial} f)$ ), then $f$ must be a holomorphic map (or antiholomorphic map) of constant curvature $4 / \operatorname{rank}(\partial f)$ (or $4 / \operatorname{rank}(\bar{\partial} f))$. When $f$ generates an orthogonal harmonic sequence and if its curvature $\kappa \geq \kappa(f)$, a number associated to the sequence generated by $f$, then $\kappa=\kappa(f)$ is constant, and $f$ is a holomorphic map which essentially generates a Frenet pseudoholomorphic sequence. We also show that for a harmonic map $f$ in a Frenet harmonic sequence of position $j$ generated by a harmonic map $g$, if its curvature $\kappa \geq \kappa(g, j)$, a number associated to the sequence and $j$, then again $\kappa=\kappa(g, j)$ is constant and the directrix $g$ is holomorphic. In particular, $f$ is a pseudoholomorphic map of constant curvature $\kappa(g, j)$. Notice that when the directrix is holomorphic and the target space is $C P^{n}$, we recover the pinching theorem of $\mathrm{J}$. Bolton, et al.

Some relations between the pinching conditions of curvature and the ranks of the $\partial$-transform and the $\bar{\partial}$-transform are discussed in this paper. As examples of using the technique developed in this paper, we prove some pinching theorems for harmonic two-spheres in $G(2,4)$ and $G(2,6)$. As a by-product, we show that for a minimal two-sphere in $G(2,4)$, if its curvature $\kappa \geq 2$, then $\kappa$ is either 2 or 4 , and all these maps are explicitly classified up to $u(4)$ congruences.

The main tool used is the method of moving frames. Extensively using this method, we are able to develop a system of partial differential equations associated to a family of invariants. The study of these invariants plays a key role in proving the main results of this paper.

Definitions and basic formulas are given in $\S 1$. General pinching theorems are proved in $\S 2$. In $\S 3$, we give a detail discussion of harmonic two-spheres in $G(2,4)$ and $G(2,6)$.

This paper is a slight revision of a part of the author's Ph.D. thesis. He would like to thank his advisor Professor Gary R. Jensen for his direction. Also, he would like to thank Janie McBane for typing this paper.

\section{HARMONIC MAPS, HARMONIC SEQUENCES AND PSEUDOHOLOMORPHIC MAPS}

The complex Grassmann manifold $G(m, N)$ is the set of all $m$-dimensional complex linear subspaces of $C^{N}$ through the origin, which can be realized as the homogeneous space $U(N) /(U(m) \times U(N-m))$, where

$$
\begin{gathered}
\pi: U(N) \rightarrow G(m, N) \\
g \rightarrow[g \cdot 0]
\end{gathered}
$$

is a principal $U(m) \times U(N-m)$-bundle, 0 is the $N \times m$ matrix

$$
\left[\begin{array}{c}
I m \\
0
\end{array}\right] \text {, }
$$

and $I_{m}$ is the $m \times m$ identity matrix. 
Let $W=\left(W_{A B}\right)=g^{-1} d g$ be the Maurer-Cartan form of $U(N)$, where $1 \leq A, B \leq N$, and let $e$ be a local section of $\pi: U(N) \rightarrow G(m, N)$. Set

$$
\Psi_{A B}=e^{*}\left(W_{A B}\right)
$$

and

$$
d s_{G(m, N)}^{2}=\sum_{\substack{1 \leq i \leq m \\ m<\alpha \leq N}} \Psi_{i \alpha} \bar{\Psi}_{i \alpha}
$$

Then $d s_{G(m, N)}^{2}$ is a $U(N)$-invariant Hermitian metric on $G(m, N)$. When $m=1$ and $N=n+1$, this is just the Fubini-Study metric on $C P^{n}$ of constant holomorphic curvature 4.

Suppose $M$ is a Riemann surface with a Riemannian metric $d s_{M}^{2}$. Then locally

$$
d s_{M}^{2}=\theta \bar{\theta}
$$

and

$$
d \theta=i w \wedge \theta
$$

where $\theta$ is a local unitary coframe of bidegree $(1,0)$ which is determined up to a $U(1)$-valued function, and $w$ is the real-valued Levi-Civita connection form associated to the coframe $\theta$. Also,

$$
d w=\frac{i}{2} \kappa \theta \wedge \bar{\theta}
$$

where $\kappa$ is the Gaussian curvature of $d s_{M}^{2}$.

Let $f$ be a smooth map from $M$ to $G(m, N)$. Choose a local unitary frame $e=\left(e_{1}, \ldots, e_{N}\right)$ along $f$ such that $e_{1}, \ldots, e_{m}$ span $f$. Then for $1 \leq A \leq N$

$$
d e_{A}=\sum_{B=1}^{n} e_{B} w_{B A},
$$

where $w_{A B}=f^{*}\left(\Psi_{A B}\right)=e^{*}\left(W_{A B}\right)$ are the entries of the pull back of the MaurerCartan form of $U(N)$ via $e$.

The Maurer-Cartan structure equations give

$$
d w_{A B}=-\sum_{c=1}^{N} w_{A C} \wedge w_{C B}
$$

and

$$
w_{A B}+\overline{w_{B A}}=0 .
$$

For $1 \leq j \leq m$ and $m+1 \leq \alpha \leq N$, set

$$
w_{\alpha j}=a_{\alpha j} \theta+b_{\alpha j} \bar{\theta} \text {. }
$$


Then $f$ is an isometric immersion if and only if

$$
\sum_{\substack{1 \leq j \leq m \\ m<\alpha x \leq N}} a_{\alpha j} b_{\alpha j}=0
$$

and

$$
\sum_{\substack{1 \leq j \leq m \\ m<\alpha \leq n}}\left(a_{\alpha j} \overline{a_{\alpha j}}+b_{\alpha \kappa j} \overline{b_{\alpha j}}\right)=1 .
$$

The harmonicity condition for $f$ is

$$
d a_{\alpha j}+i a_{\alpha j} w+\sum_{\beta=m+1}^{n} a_{\beta j} w_{\alpha \beta}-\sum_{t=1}^{m} a_{\alpha t} w_{t j} \equiv 0 \bmod \theta,
$$

which is equivalent to

$$
d b_{\alpha j}-i b_{\alpha j} w+\sum_{\beta=m+1}^{n} b_{\beta j} w_{\alpha \beta}-\sum_{t=1}^{m} b_{\alpha t} w_{t j} \equiv 0 \quad \bmod \bar{\theta} .
$$

In terms of matrix notation, we can rewrite these equations as

$$
\begin{gathered}
\operatorname{tr}\left(A^{t} \bar{B}\right)=0, \\
|A|^{2}+|B|^{2}=1, \\
d A=-i w A+A \Phi_{11}-\Phi_{22} A \bmod \theta
\end{gathered}
$$

and

$$
d B=i w B+B \Phi_{11}-\Phi_{22} B \bmod \bar{\theta}
$$

where

$$
\begin{aligned}
& A=\left(\begin{array}{ccc}
a_{m+1,1} & \cdots & a_{m+1, m} \\
\vdots & & \vdots \\
a_{N, 1} & \cdots & a_{N, m}
\end{array}\right), \quad B=\left(\begin{array}{ccc}
b_{m+1,1} & \cdots & b_{m+1, m} \\
\vdots & & \vdots \\
b_{N, 1} & \cdots & b_{N, m}
\end{array}\right), \\
& \boldsymbol{\Phi}_{11}=\left(\begin{array}{ccc}
w_{1,1} & \cdots & w_{1, m} \\
\vdots & & \vdots \\
w_{m, 1} & \cdots & w_{m, m}
\end{array}\right) \text { and } \boldsymbol{\Phi}_{22}=\left(\begin{array}{ccc}
w_{m+1, m+1} & \cdots & w_{m+1, N} \\
\vdots & & \vdots \\
w_{N, m+1} & \cdots & w_{N, N}
\end{array}\right) \text {. }
\end{aligned}
$$

Here we define the norm $|x|$ of a matrix $x$ by $|x|^{2}=\operatorname{tr}\left(x^{t} \bar{x}\right)$ in a standard way.

For a harmonic map $f$ from $M$ into $G(m, N)$, using the $\partial$-transform and the $\bar{\partial}$-transform S. S. Chern and J. G. Wolfson defined new harmonic maps, $\partial f: M \rightarrow G\left(m_{1}, N\right)$, if $f$ is not antiholomorphic, and $\bar{\partial} f: M \rightarrow G\left(m_{-1}, N\right)$, if $f$ is not holomorphic. These maps satisfy

$$
\partial f(x)=\operatorname{span}\left\{\sum_{\alpha=m+1}^{N} a_{\alpha j}(x) e_{\alpha x}(x): 1 \leq j \leq m\right\}
$$


and

$$
\bar{\partial} f(x)=\operatorname{span}\left\{\sum_{\alpha=m+1}^{N} b_{\alpha j}(x) e_{\alpha}(x): 1 \leq j \leq m\right\}
$$

for $x \in M$, except for at most isolated points. Here $m_{1}$ and $m_{-1}$ are positive integers, called the rank of $\partial f$ and the rank of $\bar{\partial} f$, respectively. Sucessively using these transforms they obtained two sequences of harmonic maps

$$
f \equiv f_{0} \stackrel{\partial}{\rightarrow} f_{1} \stackrel{\partial}{\rightarrow} f_{2} \rightarrow \cdots
$$

and

$$
f \equiv f_{0} \stackrel{\bar{\partial}}{\rightarrow} f_{-1} \stackrel{\bar{\partial}}{\rightarrow} f_{-2} \rightarrow \cdots
$$

which are called the harmonic sequences, where for $j \geq 1, f_{j} \equiv \partial f_{j-1}: M \rightarrow$ $G\left(m_{j}, N\right)$, and for $j \leq-1, f_{j} \equiv \bar{\partial} f_{j+1}: M \rightarrow G\left(m_{j}, N\right)$. We say that $f_{j}$ and $f_{i}$ are orthogonal, if for $x \in M, f_{j}(x) \perp f_{i}(x)$ as linear subspaces of $C^{N}$ with respect to the standard Hermitian inner product. If $f_{j} \perp f_{i}$ for $i \neq j$ in a sequence, we say this sequence is orthogonal. In addition, if $m_{j}$ 's are equal, we have

$$
f_{0} \underset{\bar{\partial}}{\stackrel{\partial}{\rightleftarrows}} f_{1} \underset{\bar{\partial}}{\stackrel{\partial}{\rightleftarrows}} f_{2} \underset{\bar{\partial}}{\stackrel{\partial}{\rightleftarrows}} f_{3} \ldots
$$

which is called a Frenet harmonic sequence. It is a finite sequence, and $f_{0}$ is called the directrix of this sequence.

When $f$ is holomorphic, it will generate a harmonic sequence,

$$
f \equiv f_{0} \stackrel{\partial}{\rightarrow} f_{1} \stackrel{\partial}{\rightarrow} f_{2} \rightarrow \cdots
$$

which is orthogonal and therefore is finite. This sequence is called a pseudoholomorphic sequence, and each map $f_{j}$ is called a pseudoholomorphic map generated by $f$ with position $j$.

A locally defined smooth function $V: M \supset U \rightarrow C^{L}$ is of analytic type, if either $v$ is identically zero on $U$ or for any local complex coordinate $z$ centered about $x$, the local representation of $v$ has the form $v(z)=z^{r} g(z)$ where $g(z)$ is a $C^{L}$-valued smooth function such that $g(0) \neq 0$.

The following proposition will be repeatedly used in this paper, whose proof can be found in $[\mathrm{Z}]$.

Proposition 1.1. Let $U$ be an open subset of $M, C$ be a $k \times k$-matrix-valued smooth function defined on $U$, and $\theta$ be a unitary coframe field defined on $U$. Suppose that $C$ satisfies

$$
d C=C \eta+\phi \eta-C \Psi \bmod \theta,
$$

where $\eta$ is an imaginary valued 1 -form and $\phi$ and $\Psi$ are $k \times k$-matrix-valued 1-forms with $(\operatorname{tr} \phi-\operatorname{tr} \Psi)$ imaginary valued. Then $\operatorname{det} C$ is a function of analytic type, and

$$
(\Delta \log |\operatorname{det} C|) \theta \wedge \bar{\theta}=2 k d \eta+2 d(\operatorname{tr} \phi)-2 d(\operatorname{tr} \Psi)
$$

on $U \backslash Z$, where $Z$ is the set of zeros of $\operatorname{det} C$ on $U$. 
Remark. When $k=1$ and $\phi=\Psi=0$, this is the formula given in [EGT].

2. QUANTIZATION OF CURVATURE OF HARMONIC TWO-SPHERES IN $G(m, N)$

Let $f$ be a harmonic map from a Riemann surface $M$ into $G(m, N)$. Take a local unitary frame $e=\left(e_{1}, \ldots, e_{N}\right)$ along $f$ such that $e_{1}, \ldots, e_{m}$ span $f$. Then

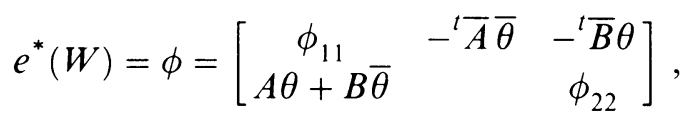

where $A, B \in C^{\infty}(M((N-m) \times m)), \phi_{11} \in C^{\infty}\left(u(m) \otimes T^{*} M\right)$ and $\phi_{22} \in$ $C^{\infty}\left(u(N-m) \otimes T^{*} M\right)$. Here we denote $C^{\infty}\left(M\left(m_{1} \times m_{2}\right)\right)$ as the set of all locally defined $m_{1} \times m_{2}$-matrix-valued smooth functions and $C^{\infty}\left(u(k) \otimes T^{*} M\right)$ as the set of all locally defined $u(k)$-valued smooth 1-forms, where $u(k)$ is the Lie algebra of $U(k)$.

The frame $e$ is determined up to $U(m) \times U(n-m)$-transformations. Changing such a local frame, $\tilde{e}=e\left[\begin{array}{cc}K_{1} & 0 \\ 0 & K_{2}\end{array}\right]$, where $K_{1} \in C^{\infty}(U(m))$ and $K_{2} \in$ $C^{\infty}(U(N-m))$, we shall have

$$
\tilde{e}^{*}(W)=\tilde{\phi}=\left[\begin{array}{cc}
\tilde{\phi}_{11} & -{ }^{t} \overline{\tilde{A}} \bar{\theta}-{ }^{t} \overline{\tilde{B}} \theta \\
\tilde{A} \theta+\tilde{B} \bar{\theta} & \tilde{\phi}_{22}
\end{array}\right]
$$

where $\tilde{A}=K_{2}^{-1} A K_{1}$ and $\tilde{B}=K_{2}^{-1} B K_{1}$. So,

$$
{ }^{t} \tilde{A} \bar{B}=\bar{K}_{1}^{-1 t} A \bar{B} \bar{K}_{1} \text {. }
$$

Setting $C={ }^{t} A \bar{B}$ and $\tilde{C}={ }^{t} \tilde{A} \bar{B}$, we see that $\operatorname{det} C=\operatorname{det} \tilde{C}$ and $\operatorname{tr} C=\operatorname{tr} \tilde{C}$. Notice that changing a local unitary coframe $\theta$ into $\tilde{\theta}, \operatorname{det} C$ becomes $u_{1} \operatorname{det} C$ and $\operatorname{tr} C$ becomes $u_{2} \operatorname{tr} C$, where $u_{1}$ and $u_{2}$ are smooth functions of absolute value 1 . Thus $|\operatorname{det} C|$ and $|\operatorname{tr} C|$ are globally defined nonnegative invariants on $M$, which are continuous.

Proposition 2.1. $\operatorname{det} C$ and $\operatorname{tr} C$ are of analytic type. Furthermore, away from zeros of $|\operatorname{det} C|$ and $|\operatorname{tr} C|$, they satisfy

$$
\Delta \log |\operatorname{det} C|=2 m \kappa
$$

and

$$
\Delta \log |\operatorname{tr} C|=2 \kappa,
$$

where $\kappa$ is the Gaussian curvature of the metric $d s_{M}^{2}$.

Proof. Taking the exterior derivative of $C$ and using (1.12) and (1.13) we obtain

$$
d C=-2 i w C+{ }^{t} \phi_{11} C-C^{t} \phi_{11} \bmod \theta .
$$

Applying Proposition 1.1 to (2.4),

$$
\Delta \log |\operatorname{det} C| \theta \wedge \bar{\theta}=-4 m i d w .
$$

(2.2) then follows from using (1.5) to (2.5). 
For (2.3), we take the trace on both sides of (2.4). Then

$$
d(\operatorname{tr} C)=-2 i w(\operatorname{tr} C) \bmod \theta,
$$

and the conclusion again is obtained by using (1.5) and Proposition 1.1 for the case of $k=1$. Q.E.D.

Following this proposition, we have

Corollary 2.1 (Vanishing Theorem). If $M$ is a closed Riemann surface, then either the genus $g$ of $M$ is positive or both $|\operatorname{det} c|$ and $|\operatorname{tr} c|$ vanish identically. In particular, if the Gaussian curvature $\kappa \geq 0$, then either $\kappa=0$ or both $|\operatorname{det} c|$ and $|\operatorname{tr} c|$ are identically zero.

Proof. Use the Gauss-Bonnet formula and Stokes' Theorem on (2.2) and (2.3). Q.E.D.

Remarks. 1. Corollary 2.1 is known [CW].

2. When $M=S^{2}$, then $|\operatorname{tr} C|=0$, which means that harmonic maps from $S^{2}$ into $G(m, N)$ are weakly conformal (i.e., (1.10) holds).

We say that the $\partial$-transform (resp. the $\bar{\partial}$-transform) is degenerate, if $\operatorname{rank}(\partial f)<\operatorname{rank}(f)(\operatorname{resp} . \operatorname{rank}(\bar{\partial} f)<\operatorname{rank}(f))$.

Corollary 2.2. For a harmonic map from $S^{2}$ into $G(m, 2 m)$, one of the $\partial$ transform and the $\bar{\partial}$-transform must be degenerate.

Proof. Observe that in this case $\operatorname{det} C=\operatorname{det} A \cdot \operatorname{det} \bar{B}$, and generically $\operatorname{rank}(A)$ $=\operatorname{rank}(\partial f)$ and $\operatorname{rank}(B)=\operatorname{rank}(\bar{\partial} f)$. Q.E.D.

Now suppose that $M=S^{2}$ is the Riemann sphere and $f$ is a harmonic isometric immersion so that $\kappa$ is the Gaussian curvature of the induced metric $f^{*} d s_{G(m, N)}^{2}$. The following theorem says that if the curvature is large enough, then $f$ is forced to be holomorphic or antiholomorphic.

Theorem 2.1. If $f$ is not antiholomorphic, and if $\kappa \geq 4 / \operatorname{rank}(\partial f)$, then $f$ is a holomorphic map of constant curvature $4 / \operatorname{rank}(\partial f)$. Similarly, if $f$ is not holomorphic, and if $\kappa \geq 4 / \operatorname{rank}(\bar{\partial} f)$, then $f$ is an antiholomorphic map of constant curvature $4 / \operatorname{rank}(\bar{\partial} f)$.

Proof. Suppose $f$ is not antiholomorphic. Choose a local unitary frame $e=$ $\left(e_{1}, \ldots, e_{N}\right)$ along $f$ so that $e_{1}, \ldots, e_{m}$ span $f$ and $e_{m+1}, \ldots, e_{m+k^{0}}$ span $\partial f$, where $k_{0}=\operatorname{rank}(\partial f)$. Furthermore, we can require that $e_{k_{0}+1}, \ldots, e_{m}$ span the kernel of the $\partial$-transform. The pull back of the Maurer-Cartan form of $U(N)$ by $e$ is then

$$
\phi=\left[\begin{array}{lccc}
\Omega_{11} & \Omega_{12} & -{ }^{\tau} \bar{A}_{11} \bar{\theta}-{ }^{\tau} \bar{B}_{11} \theta & -{ }^{\tau} \bar{B}_{21} \theta \\
\Omega_{21} & \Omega_{22} & -{ }^{\tau} \bar{B}_{12} \theta & -{ }^{\tau} \bar{B}_{22} \theta \\
A_{11} \theta+B_{11} \bar{\theta} & B_{12} \bar{\theta} & \Omega_{33} & \Omega_{34} \\
B_{21} \bar{\theta} & B_{22} \bar{\theta} & \Omega_{43} & \Omega_{44}
\end{array}\right],
$$

where $A_{11}, B_{11} \in C^{\infty}\left(u\left(k_{0} \times k_{0}\right)\right), B_{21} \in C^{\infty}\left(M\left(\left(N-m-k_{0}\right) \times k_{0}\right)\right), B_{12} \in$ $C^{\infty}\left(M\left(k_{0} \times\left(m-k_{0}\right)\right)\right), \quad B_{22} \in C^{\infty}\left(M\left(m-k_{0}\right) \times\left(m-k_{0}\right)\right), \Omega_{11}, \Omega_{33} \in$ 
$C^{\infty}\left(u\left(k_{0}\right) \otimes T^{*} S^{2}\right), \Omega_{21} \in C^{\infty}\left(M\left(\left(m-k_{0}\right) \times k_{0}\right) \otimes T^{*} S^{2}\right), \Omega_{12}=-{ }^{\tau} \bar{\Omega}_{21}$, $\Omega_{22} \in C^{\infty}\left(u\left(m-k_{0}\right) \otimes T^{*} S^{2}\right), \Omega_{43} \in C^{\infty}\left(M\left(\left(N-m-k_{0}\right) \times k_{0}\right) \otimes T^{*} S^{2}\right)$, $\Omega_{34}=-{ }^{\tau} \bar{\Omega}_{43}$ and $\Omega_{44} \in C^{\infty}\left(u\left(N-m-k_{0}\right) \otimes T^{*} S^{2}\right)$. From $\left(1.11^{\prime}\right)$, we have

$$
\left|A_{11}\right|^{2}+\left|B_{11}\right|^{2}+\left|B_{12}\right|^{2}+\left|B_{21}\right|^{2}+\left|B_{22}\right|^{2}=1 \text {. }
$$

It can be easily checked that $\left|\operatorname{det} A_{11}\right|$ is a well-defined invariant on $S^{2}$. Harmonicity condition $\left(1.12^{\prime}\right)$ of $f$ gives

$$
\begin{aligned}
d\left[\begin{array}{cc}
A_{11} & 0 \\
0 & 0
\end{array}\right]= & -i w\left[\begin{array}{cc}
A_{11} & 0 \\
0 & 0
\end{array}\right]+\left[\begin{array}{cc}
A_{11} & 0 \\
0 & 0
\end{array}\right]\left[\begin{array}{ll}
\Omega_{11} & \Omega_{12} \\
\Omega_{21} & \Omega_{22}
\end{array}\right] \\
& -\left[\begin{array}{ll}
\Omega_{33} & \Omega_{34} \\
\Omega_{43} & \Omega_{44}
\end{array}\right]\left[\begin{array}{cc}
A_{11} & 0 \\
0 & 0
\end{array}\right] \bmod \theta,
\end{aligned}
$$

from which it follows that

$$
\begin{gathered}
d A_{11}=-i w A_{11}+A_{11} \Omega_{11}-\Omega_{33} A_{11} \bmod \theta \\
\Omega_{43} A_{11}=0 \bmod \theta
\end{gathered}
$$

and

$$
A_{11} \Omega_{12}=0 \bmod \theta .
$$

(2.10) shows that $\Omega_{12}$ and $\Omega_{43}$ are matrices of bidegree (1.0) forms. So

$$
\Omega_{12}=X_{12} \theta
$$

and

$$
\Omega_{43}=X_{43} \theta,
$$

where $X_{12}$ and $X_{43}$ are matrix-valued smooth functions.

Applying Proposition 1.1 to (2.9) and using (1.5), we get

$$
\Delta \log \left|\operatorname{det} A_{11}\right| \theta \wedge \bar{\theta}=k_{0} \kappa \theta \wedge \bar{\theta}+2 d\left(\operatorname{tr} \Omega_{11}-\operatorname{tr} \Omega_{33}\right) .
$$

By (1.7), (2.7), (2.10) and (2.11),

(2.14) $\Delta \log \left|\operatorname{det} A_{11}\right|$

$$
\begin{aligned}
& =k_{0} \kappa+2\left(\left|X_{12}\right|^{2}+\left|X_{43}\right|^{2}-2\left|A_{11}\right|^{2}+2\left|B_{11}\right|^{2}+\left|B_{12}\right|^{2}+\left|B_{21}\right|^{2}\right) \\
& \geq k_{0} \kappa-4 .
\end{aligned}
$$

Since $\kappa \geq 4 / k_{0}$,

$$
\Delta \log \left|\operatorname{det} A_{11}\right| \geq 0 .
$$

Notice that (2.15) holds on $S^{2}$ except for at most finitely many points, and that $\left|\operatorname{det} A_{11}\right|$ assumes its positive maximum value at some point where (2.15) is valid. By the maximum principle of subharmonic functions, it therefore must be constant. Then by (2.7) and (2.14) again, $\kappa=4 / k_{0}$ and $B_{11}=B_{12}=B_{21}=$ $B_{22}=0$, which shows that $f$ is a holomorphic map of constant curvature $4 / k_{0}$. 
A similar argument will work for proving the second claim of the statement. Q.E.D.

Recall that a holomorphic map or an antiholomorphic map from the Riemann sphere into a complex Grassmann manifold is always stable. Thus we have

Corollary 2.3. If $\operatorname{rank}(\partial f) \neq 0$ (resp. $\operatorname{rank}(\bar{\partial} f) \neq 0$, and if $\kappa \geq 4 / \operatorname{rank}(\partial f)$ (resp. $K \geq 4 / \operatorname{rank}(\bar{\partial} f)$ ), then $f$ is stable.

Remark. One can construct a holomorphic map from $S^{2}$ into $G(m, N)$ with $\operatorname{rank}(\partial f)=k_{0}$ and constant curvature $\kappa=4 / k_{0}$ in terms of homogeneous coordinates in the following way,

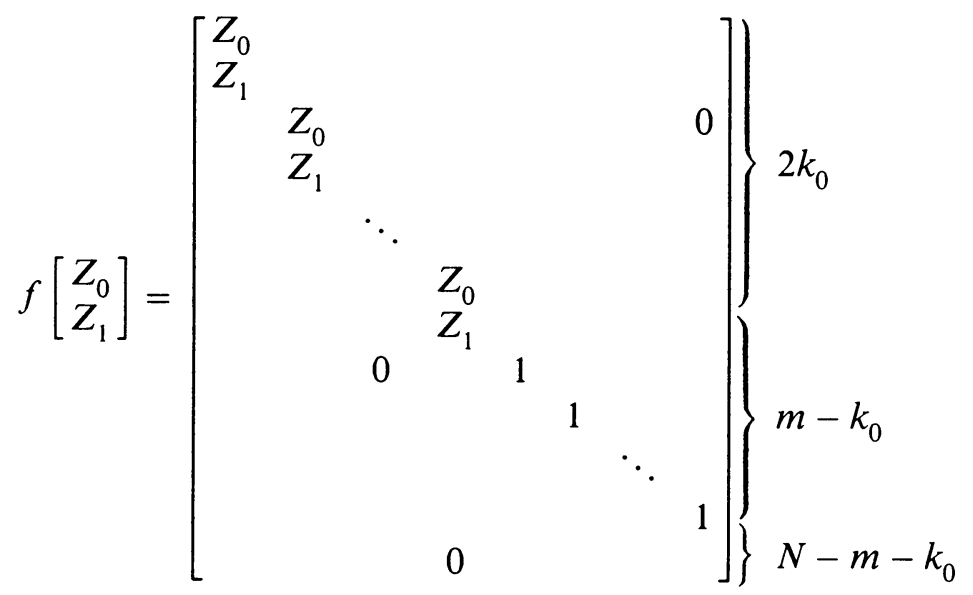

When $k_{0}>0$, a small perturbation of this map will produce a holomorphic curve of nonconstant curvature $\kappa$ with $\operatorname{rank}(\partial f)=k_{0}$ and arbitrarily small positive value of $\left|\kappa-4 / k_{0}\right|$.

For example, for any $\varepsilon>0$ one can define $f_{\varepsilon}: S^{2} \rightarrow G(m, N)$ by

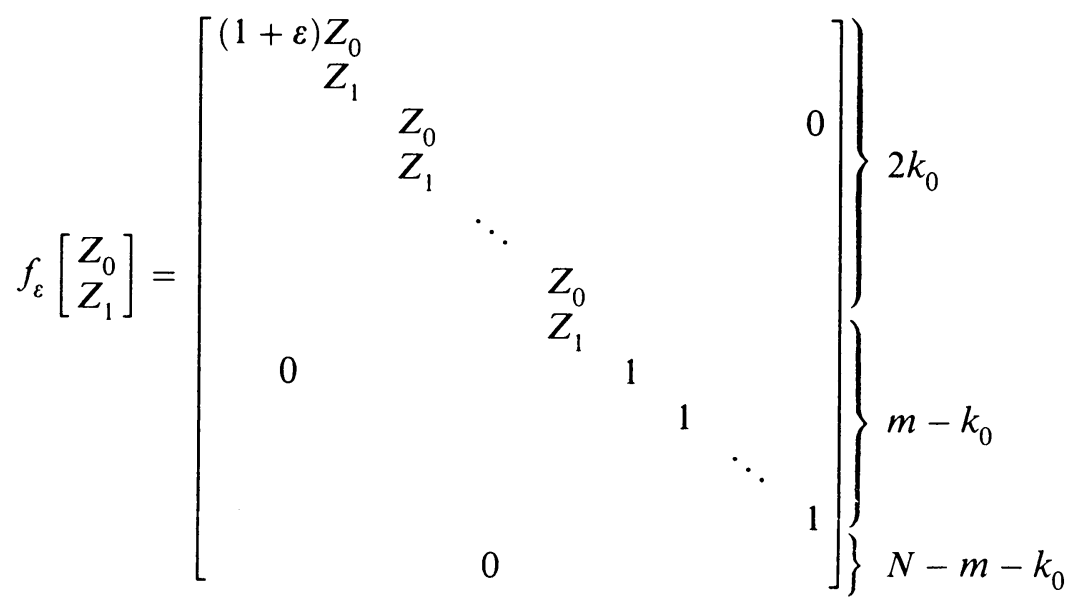


It is holomorphic. In terms of the complex coordinate $Z$ on $S^{2}$, the induced metric is $d s^{2}=h(Z)|d z|^{2}$, where

$$
h(Z)=\frac{k_{0}-1}{\left(1+|Z|^{2}\right)^{2}}+\frac{(1+\varepsilon)^{2}}{\left(|Z|^{2}+(1+\varepsilon)^{2}\right)^{2}} .
$$

Its curvature is

$$
\begin{aligned}
\kappa=\frac{4}{h^{3}(Z)}\left[\frac{\left(k_{0}-1\right)^{2}}{\left.1+|Z|^{2}\right)^{6}}+\frac{(1+\varepsilon)^{6}}{\left(|Z|^{2}+(1+\varepsilon)^{2}\right)^{6}}\right. \\
+\frac{\left(k_{0}-1\right)(1+\varepsilon)^{4}}{\left(1+|Z|^{2}\right)^{2}\left(|Z|^{2}+(1+\varepsilon)^{2}\right)^{4}} \\
+\frac{\left(k_{0}-1\right)(1+\varepsilon)^{2}}{\left(1+|Z|^{2}\right)^{4}\left(|Z|^{2}+(1+\varepsilon)^{2}\right)^{2}} \\
\\
\left.\quad-\frac{2\left(k_{0}-1\right)(1+\varepsilon)^{2}|Z|^{2}(2 \varepsilon+\varepsilon)^{2}}{\left(1+|Z|^{2}\right)^{4}\left(|Z|^{2}+(1+\varepsilon)^{2}\right)^{4}}\right],
\end{aligned}
$$

which is not constant. Since $\lim _{\varepsilon \rightarrow 0} f_{\varepsilon}=f$, one certainly can make $\left|\kappa-4 / k_{0}\right|$ as small as desired.

Now, if the curvature $\kappa$ is not that big, the following theorem describes some relations between the pinching conditions of the curvature $\kappa$ and the ranks of the $\partial$-transform and the $\bar{\partial}$-transform.

Theorem 2.2. Let $f$ be a harmonic isometric immersion from $S^{2}$ into $G(m, N)$, where $N \geq 2 m$. Let $k_{1}=\operatorname{rank}(\partial f), k_{2}=\operatorname{rank}(\bar{\partial} f)$ and $\kappa$ be the Gaussian curvature. We have the following.

(i) If $0<k_{1}+k_{2} \leq m$ and $\kappa \geq 4 /\left(k_{1}+k_{2}\right)$, then $\kappa=4 /\left(k_{1}+k_{2}\right)$ is constant, and $f=\left[f_{1} \oplus f_{2} \oplus V\right]: S^{2} \rightarrow G(m, n)$, where $f_{1} \perp f_{2} \perp V$ such that $f_{1}: S^{2} \rightarrow G\left(k_{1}, N\right)$ is holomorphic, $f_{2}: S^{2} \rightarrow G\left(k_{2}, N\right)$ is antiholomorphic and $V$ is a constant complex vector subspace of $C^{N}$ of dimension $m-k_{1}-k_{2}$.

(ii) If $0<k_{1}<m, 0<k_{2}<m$ and $k_{1}+k_{2}>m$, then $\min \kappa<4 /\left(k_{1}+k_{2}\right)$.

(iii) If $k_{1}=m$ and $0<k_{2}<m$ (or $0<k_{1}<m$ and $k_{2}=m$ ) when $N>2 m$, then $\min \kappa<8 /\left(2 k_{1}+3 k_{2}\right) ;\left(\right.$ or $\left.\min \kappa<8 /\left(2 k_{2}+3 k_{1}\right)\right)$; when $N=2 m$, then $\min \kappa<4 /\left(k_{1}+2 k_{2}\right)\left(\right.$ or $\left.\min \kappa<4 /\left(k_{2}+2 k_{1}\right)\right)$.

(iv) If $k_{1}=k_{2}=m$ and $\kappa \geq 1 / m$, then $\kappa=1 / m$ is constant and $\partial f \perp \bar{\partial} f$. Furthermore, $f$ is a pseudoholomorphic map in the following sequence,

$$
f_{-1} \stackrel{\dot{b}}{\rightarrow} f \equiv f_{0} \stackrel{\dot{\partial}}{\rightarrow} f_{1},
$$

where $f_{-1}: S^{2} \rightarrow G(m, N)$ is holomorphic and $f_{1}: S^{2} \rightarrow G(m, N)$ is antiholomorphic.

Remarks. 1. The hypothesis that $N \geq 2 m$ is not crucial, since for any harmonic map $f$, its orthogonal complement, denoted by $f^{\perp}$, is also a harmonic map having the same induced metric as that of $f$. Moreover, $\operatorname{rank}(\partial f)$ 
$=\operatorname{rank}\left(\bar{\partial}\left(f^{\perp}\right)\right)$ and $\operatorname{rank}(\bar{\partial} f)=\operatorname{rank}\left(\partial\left(f^{\perp}\right)\right)$. So we can replace $f$ by $f^{\perp}$ if it is necessary.

2. (iii) says that when $N>2 m$, if $\kappa \geq 8 /(2 m+3 \operatorname{rank}(\bar{\partial} f)$ ) (or $\kappa \geq$ $8 /(2 m+3 \operatorname{rank}(\partial f)))$, then the $\partial$-transform (or $\bar{\partial}$-transform) must be degenerate; when $N=2 m$, if $\kappa \geq 4 /(m+2 \operatorname{rank}(\bar{\partial} f))($ or $\kappa \geq 4 /(m+2 \operatorname{rank}(\partial f)))$, then the $\partial$-transform (or $\bar{\partial}$-transform) must be degenerate.

Proof. Choosing a unitary frame $e$ as in the proof of Theorem 2.1 and adopting the notations there we have

$$
\begin{aligned}
\Delta \log \left|\operatorname{det} A_{11}\right|=k_{1} \kappa+2 & \left(\left|X_{12}\right|^{2}+\left|X_{43}\right|^{2}-2\left|A_{11}\right|^{2}\right. \\
& \left.+2\left|B_{11}\right|^{2}+\left|B_{12}\right|^{2}+\left|B_{21}\right|^{2}\right) .
\end{aligned}
$$

Change such a unitary frame $e$ into $\tilde{e}$ such that $\tilde{e}=\left(\tilde{e}_{1}, \tilde{e}_{2}, \ldots, \tilde{e}_{N}\right)$, where $\tilde{e}_{1}, \ldots, \tilde{e}_{m} \operatorname{span} f, \tilde{e}_{m+1}, \ldots, \tilde{e}_{m+k_{2}}$ span $\bar{\partial} f$ and $\tilde{e}_{k_{2}+1}, \ldots, \tilde{e}_{m}$ span the kernel of the $\bar{\partial}$-transform. If we denote the corresponding new quantities by adding tildes, then a similar argument leads to

$$
\begin{aligned}
& \Delta \log \left|\operatorname{det} \tilde{B}_{11}\right|=k_{2} \kappa+2\left(\left|\tilde{X}_{12}\right|^{2}+2\left|\tilde{A}_{11}\right|^{2}+\left|\tilde{A}_{21}\right|^{2}\right. \\
&\left.-2\left|\tilde{B}_{11}\right|^{2}+\left|\tilde{A}_{12}\right|^{2}+\left|\tilde{X}_{43}\right|^{2}\right)
\end{aligned}
$$

Since $\tilde{e}=\left[\begin{array}{cc}K_{1} & 0 \\ 0 & K_{2}\end{array}\right] e$, where $K_{1} \in C^{\infty}(U(m))$ and $K_{2} \in C^{\infty}(U(N-m))$, we have $\tilde{A}=K_{2}^{-1} A K_{1}$ and $\tilde{B}=K_{2}^{-1} B K_{1}$, and therefore $|\tilde{A}|^{2}=|A|^{2}$ and $|\tilde{B}|^{2}=|B|^{2}$.

If $0<k_{1}+k_{2} \leq m$ and $\kappa \geq 4 /\left(k_{1}+k_{2}\right)$, then combining (2.16) and (2.17), together with $\left(1.11^{\prime}\right)$, we shall have

$\Delta \log \left(\left|\operatorname{det} A_{11}\right|\left|\operatorname{det} \tilde{B}_{11}\right|\right)$

$$
\begin{aligned}
& =\left(k_{1}+k_{2}\right) \kappa+2\left(\left|X_{12}\right|^{2}+\left|\tilde{X}_{12}\right|^{2}+\left|X_{43}\right|^{2}+\left|\tilde{X}_{43}\right|^{2}-2\left|A_{11}\right|^{2}-2\left|\tilde{B}_{11}\right|^{2}\right. \\
& \left.\quad \quad+2\left|B_{11}\right|^{2}+\left|B_{12}\right|^{2}+\left|B_{21}\right|^{2}+2\left|\tilde{A}_{11}\right|^{2}+\left|\tilde{A}_{12}\right|^{2}+\left|\tilde{A}_{21}\right|^{2}\right) \\
& \quad\left(k_{1}+k_{2}\right) \kappa-4\left(\left|A_{11}\right|^{2}+\left|\tilde{B}_{11}\right|^{2}\right) \\
& =\left(k_{1}+k_{2}\right) \kappa-4 \geq 0 .
\end{aligned}
$$

Using the same argument as in the proof of Theorem 2.1 , we see that the globally defined invariant $\left|\operatorname{det} A_{11}\right|\left|\operatorname{det} \tilde{B}_{11}\right|$ is constant and $\kappa=4 /\left(k_{1}+k_{2}\right)$ is constant. Moreover, (2.18) implies

$$
\left|X_{12}\right|=\left|X_{43}\right|=\left|B_{11}\right|=\left|B_{12}\right|=\left|B_{21}\right|=0,
$$

and

$$
\left|\tilde{X}_{12}\right|=\left|\tilde{X}_{43}\right|=\left|\tilde{A}_{11}\right|=\left|\tilde{A}_{12}\right|=\left|\tilde{A}_{21}\right|=0
$$

which mean that $\partial f \perp \bar{\partial} f$. 
Specifying the local frame $e$ further by requiring that $e_{k_{1}+k_{2}+1}, \ldots, e_{m}$ span the kernel of the $\bar{\partial}$-transform and using (2.19), the pull back of the MaurerCartan form by $e$ is then of the form

$$
\phi=\left[\begin{array}{cccccc}
\phi_{1} & 0 & 0 & -{ }^{\tau} \bar{A}_{11} \bar{\theta} & 0 & 0 \\
0 & \phi_{2} & 0 & 0 & -{ }^{\tau} \bar{B}_{22} \theta & 0 \\
0 & 0 & \phi_{3} & 0 & 0 & 0 \\
A_{11} \theta & 0 & 0 & \phi_{4} & 0 & 0 \\
0 & B_{22} \bar{\theta} & 0 & 0 & \phi_{5} & 0 \\
0 & 0 & 0 & 0 & 0 & \phi_{6}
\end{array}\right]
$$

where $\phi_{i}, i=1, \ldots, 6$, are matrix-valued 1-forms, $A_{11} \in C^{\infty}\left(M\left(k_{1} \times k_{1}\right)\right)$ and $B_{22} \in C^{\infty}\left(M\left(k_{2} \times k_{2}\right)\right)$.

Now, we see that $f=\left[f_{1} \oplus f_{2} \oplus V\right]$, where $f_{1} \perp f_{2} \perp V$, and $f_{1}=$ $\operatorname{span}\left\{e_{1}, e_{2}, \ldots, e_{k_{1}}\right\}: S^{2} \rightarrow G\left(k_{1}, N\right)$ is holomorphic,

$$
f_{2}=\operatorname{span}\left\{e_{k_{1}+1}, \ldots, e_{k_{1}+k_{2}}\right\}: S^{2} \rightarrow G\left(k_{2}, N\right)
$$

is antiholomorphic and $V=\operatorname{span}\left\{e_{k_{1}+k_{2}+1}, \ldots, e_{m}\right\}$ is a constant vector subspace of complex dimension $m-k_{1}-k_{2}$.

Suppose now $0<k_{1}<m, 0<k_{2}<m$ and $m<k_{1}+k_{2}$. If $\min \kappa \geq$ $4 /\left(k_{1}+k_{2}\right)$, then (2.19) would imply $f=\left[g_{1} \oplus g_{2}\right]$, where $g_{1} \perp g_{2}, g_{1}: S^{2} \rightarrow$ $G\left(k_{1}, N\right)$ is holomorphic and $g_{2}: S^{2} \rightarrow G\left(m-k_{1}, N\right)$ is antiholomorphic, with $\operatorname{rank}\left(\bar{\partial} g_{2}\right)=k_{2}$. But this means that $m-k_{1} \geq k_{2}$ which contradicts the hypothesis that $k_{1}+k_{2}>m$.

When $k_{1}=m$ and $0<k_{2}<m$ (or $0<k_{1}<m$ and $k_{2}=m$ ), there are two cases.

(a) $N>2 m$. In this case, since $k_{1}=m, X_{12}=B_{12}=B_{22}=0$, and $|B|^{2}=\left|B_{11}\right|^{2}+\left|B_{21}\right|^{2}$. Thus (2.16) becomes

$$
\begin{aligned}
\Delta \log \left|\operatorname{det} A_{11}\right| & =k_{1} \kappa+2\left(\left|X_{43}\right|^{2}-2\left|A_{11}\right|^{2}+2\left|B_{11}\right|^{2}+\left|B_{21}\right|^{2}\right) \\
& \geq k_{1} \kappa+2\left(-2|A|^{2}+|B|^{2}\right) .
\end{aligned}
$$

Combining it with (2.17), we get

$$
\begin{aligned}
\Delta \log \left|\operatorname{det} A_{11}\right|^{2}\left|\operatorname{det} \tilde{B}_{11}\right|^{3} & \geq\left(2 k_{1}+3 k_{2}\right) \kappa-8\left(|A|^{2}+|B|^{2}\right) \\
& =\left(2 k_{1}+3 k_{2}\right) \kappa-8 .
\end{aligned}
$$

If $\min k \geq 8 /\left(2 k_{1}+3 k_{2}\right)$, then it would follow by (2.17) and (2.21) that $\left|\tilde{A}_{11}\right|=\left|\tilde{A}_{12}\right|=\left|\tilde{A}_{21}\right|=\left|\tilde{X}_{12}\right|=0$, which means that $\operatorname{rank}(\tilde{A})=\operatorname{rank}(\partial f)<m$, contradicting the assumption on rank $\partial f$.

(b) $N=2 m$. In this case, $X_{12}=X_{34}=B_{12}=B_{21}=B_{22}=0$ and $|B|^{2}=$ $\left|B_{11}\right|^{2}$. So (2.16) is now

$$
\Delta \log \left|\operatorname{det} A_{11}\right|=k_{1} \kappa+4\left(|B|^{2}-\left|A_{11}\right|^{2}\right),
$$


from which, together with (2.17), it follows that

$$
\Delta \log \left(\left|\operatorname{det} A_{11}\right|\left|\operatorname{det} \tilde{B}_{11}\right|^{2}\right) \geq\left(k_{1}+2 k_{2}\right) \kappa-4 \text {. }
$$

If $\min \kappa \geq 4 /\left(k_{1}+k_{2}\right)$, then again $\operatorname{rank}(\partial f)<m$, which is a contradiction.

Finally, suppose $k_{1}=k_{2}=m$ and $\kappa=1 / m$. (2.16) and (2.17) become

$$
\begin{aligned}
\Delta \log \left|\operatorname{det} A_{11}\right| & =k_{1} \kappa+2\left(\left|X_{43}\right|^{2}-2\left|A_{11}\right|^{2}+|B|^{2}+\left|B_{11}\right|^{2}\right) \\
& \geq m \kappa-4|A|^{2}+2|B|^{2}
\end{aligned}
$$

and

$$
\begin{aligned}
\Delta \log \left|\operatorname{det} \tilde{B}_{11}\right| & =k_{2} \kappa+2\left(\left|\tilde{X}_{43}\right|^{2}-2\left|\tilde{B}_{11}\right|^{2}+|\tilde{A}|^{2}+\left|\tilde{A}_{11}\right|^{2}\right) . \\
& \geq m \kappa-4|B|^{2}+2|A|^{2} .
\end{aligned}
$$

So

$$
\Delta \log \left(\left|\operatorname{det} A_{11}\right|\left|\operatorname{det} \tilde{B}_{11}\right|\right) \geq 2 m \kappa-2 \geq 0 .
$$

Thus $\kappa=1 / m$ is constant, and $X_{43}=B_{11}=\tilde{X}_{43}=\tilde{A}_{11}=0$. Now it is easy to see that $\partial f \perp \bar{\partial} f$ and

$$
f_{-1} \equiv \bar{\partial} f \stackrel{\partial}{\rightarrow} f \equiv f_{0} \stackrel{\partial}{\rightarrow} f_{1} \equiv \partial f,
$$

where $f_{-1}$ is holomorphic and $f_{1}$ is antiholomorphic. Q.E.D.

An interesting situation is when the harmonic map $f$ generates an orthogonal harmonic sequence,

$$
f \equiv f_{0} \stackrel{\partial}{\rightarrow} f_{1} \stackrel{\partial}{\rightarrow} f_{2} \rightarrow \cdots \stackrel{\partial}{\rightarrow} f_{n},
$$

such that: when $0 \leq j \leq k_{0}$, then the rank $\left(f_{j}\right)=m_{0}$; and for $1 \leq t \leq s$, when

$$
\sum_{i=0}^{t-1} k_{i}+1 \leq j \leq \sum_{i=0}^{t} k_{i}
$$

then the $\operatorname{rank}\left(f_{j}\right)=m_{t} ;$ and $\sum_{i=0}^{s} k_{i}=n$.

For such a map, we can prove the following theorem.

Theorem 2.3. Let $f$ be a harmonic isometric immersion from $S^{2}$ into $G(m, N)$. Suppose that $f$ generates an orthogonal harmonic sequence as above, and the Gaussian curvature $\kappa$ is bounded below by the constant

$$
\kappa(f)=\frac{4 \prod_{j=0}^{s}\left(k_{j}+1\right)}{\sum_{r=0}^{s} \prod_{j=r}^{s} k_{r}\left(k_{j}+1\right) m_{r}} .
$$

Then $k=\kappa(f)$ and $f$ is a holomorphic map which essentially generates a Frenet pseudoholomorphic sequence.

Proof. Suitably choose a smooth unitary frame $e$ so that the pull back of the Maurer-Cartan form via $e$ is

$$
\phi=\left[\begin{array}{ccccccccc}
\phi_{0} & -{ }^{\tau} \bar{A}_{0} \bar{\theta} & & & 0 & & & -{ }^{\tau} \bar{B}_{1} \theta & -{ }^{\tau} \bar{B}_{2} \theta \\
A_{0} \theta & \phi_{1} & -{ }^{\tau} \bar{A}_{1} \bar{\theta} & & & & & & \\
& 0 & & A_{j-1} \theta & \phi_{j} & -{ }^{\tau} \bar{A}_{j} \bar{\theta} & 0 & \\
B_{1} \bar{\theta} & & & & & & A_{n-1} \theta & \phi_{n} & -{ }^{\tau} \bar{A}_{n} \bar{\theta} \\
B_{2} \bar{\theta} & & & 0 & & & & A_{n} \theta & \phi_{n+1}
\end{array}\right]
$$


where $B_{1} \in C^{\infty}\left(M\left(m_{s} \times m_{0}\right)\right), B_{2} \in C^{\infty}\left(M\left(\left(N-\sum_{i=0}^{s} k_{i} m_{i}-m_{0}\right) \times m_{0}\right)\right)$; for each $r=0, \ldots, s$, when $k_{-1}+\cdots+k_{r-1}<j<k_{-1}+\cdots+k_{r}, A_{j} \in$ $C^{\infty}\left(M\left(m_{r} \times m_{r}\right)\right):$ for $r=0, \ldots, s-1, A_{k_{0}+\cdots+k_{r}} \in C^{\infty}\left(M\left(m_{r} \times m_{r+1}\right)\right)$; $A_{n} \in C^{\infty}\left(M\left(\left(n-\sum_{i=0}^{s} k_{i} m_{i}-m_{0}\right) \times m_{s}\right)\right)$; for $r=0, \ldots, s$, when $k_{-1}$ $+\cdots+k_{r-1}<j<k_{-1}+\cdots+k_{r}, \phi_{j} \in C^{\infty}\left(u\left(m_{r}\right) \otimes T^{*} S^{2}\right)$ and $\phi_{s+1} \in$ $C^{\infty}\left(u\left(N-\sum_{i=0}^{s} k_{i} m_{i}-m_{0}\right) \otimes T^{*} S^{2}\right)$. Here we set $k_{-1}=0$ for notational convenience. Also, we can require

$$
A_{k_{0}+\cdots+k_{r}}=\left[0, \tilde{A}_{k_{0}+\cdots+k_{r}}\right]
$$

where $\tilde{A}_{k_{0}+\cdots+k_{r}} \in C^{\infty}\left(M\left(m_{r+1} \times m_{r+1}\right)\right)$. Now $\left(1.11^{\prime}\right)$ becomes

$$
\left|A_{0}\right|^{2}+\left|B_{1}\right|^{2}+\left|B_{2}\right|^{2}=1
$$

and the harmonicity condition $\left(1.12^{\prime}\right)$ and the Maurer-Cartan structure equations imply

$$
d A_{j}=-i w A_{j}+A_{j} \phi_{j}-\phi_{j+1} A_{j} \bmod \theta,
$$

for $j=0, \ldots, n-1$. Thus, for $k_{-1}+\cdots+k_{r-1}<j<k_{-1}+\cdots+k_{r}$, where $r=0, \ldots, s$,

$$
\Delta \log \left|\operatorname{det} A_{j}\right| \theta \wedge \bar{\theta}=2 d\left(-i m_{r} w+\operatorname{tr} \phi_{j}-\operatorname{tr} \phi_{j+1}\right),
$$

and so

$$
\Delta \log \left|\operatorname{det} A_{j}\right|=m_{r} \kappa+2\left(\left|A_{j-1}\right|^{2}+\left|A_{j+1}\right|^{2}-2\left|A_{j}\right|^{2}\right),
$$

if $j \neq n-1,0$,

$$
\Delta \log \left|\operatorname{det} A_{n-1}\right|=m_{s} \kappa+2\left(\left|A_{n-2}\right|^{2}+\left|A_{n}\right|^{2}-2\left|A_{n-1}\right|^{2}+\left|B_{1}\right|^{2}\right)
$$

and

$$
\begin{aligned}
\Delta \log \left|\operatorname{det} A_{0}\right| & =m_{0} \kappa+2\left(\left|A_{1}\right|^{2}-2\left|A_{0}\right|^{2}+\left|B_{1}\right|^{2}+\left|B_{2}\right|^{2}\right) \\
& \geq m_{0} \kappa+2\left(\left|A_{1}\right|^{2}-2\left|A_{0}\right|^{2}\right),
\end{aligned}
$$

if $k_{0} \neq 0$.

For $j=k_{0}+\cdots+k_{r}$, set

$$
\phi_{j}=\left[\begin{array}{ll}
\Omega_{11}^{j} & \Omega_{12}^{j} \\
\Omega_{21}^{j} & \Omega_{22}^{j}
\end{array}\right],
$$

where $\Omega_{11}^{j} \in C^{\infty}\left(\mu\left(m_{r}-m_{r+1}\right) \otimes T^{*} S^{2}\right), \Omega_{22}^{j} \in C^{\infty}\left(\mu\left(m_{r+1}\right) \otimes T^{*} S^{2}\right)$ and $\Omega_{21}^{j}=-{ }^{\tau} \overline{\Omega_{12}^{j}} \in C^{\infty}\left(M\left(m_{r+1} \times\left(m_{r}-m_{r+1}\right)\right) \otimes T^{*} S^{2}\right)$. Set also

$$
A_{j-1}=\left[\begin{array}{c}
D_{1}^{j-1} \\
D_{2}^{j-1}
\end{array}\right] \text {, }
$$

where $D_{1}^{j-1} \in C^{\infty}\left(M\left(\left(m_{r}-m_{r+1}\right) \times m_{r}\right)\right)$ and $D_{2}^{j-1} \in C^{\infty}\left(M\left(m_{r+1} \times m_{r}\right)\right)$. Then

$$
d \tilde{A}_{j}=-i w \tilde{A}_{j}+\tilde{A}_{j} \Omega_{22}^{j}-\phi_{j+1} \tilde{A}_{j} \bmod \theta
$$


and

$$
\tilde{A}_{j} \Omega_{21}^{j}=0 \bmod \theta,
$$

from which it follows that

$$
\Omega_{21}^{j}=X_{21}^{j} \theta
$$

$$
\begin{aligned}
\Delta \log \left|\operatorname{det} \tilde{A}_{k_{0}+\cdots+k_{r}}\right|=m_{r+1} \kappa+2\left(\left|D_{2}^{k_{0}+\cdots+k_{r}-1}\right|^{2}+\left|X_{2}^{k_{0}+\cdots+k_{r}}\right|^{2}\right. \\
\left.-2\left|\tilde{A}_{k_{0}+\cdots+k_{r}}\right|^{2}+\left|A_{k_{0}+\cdots+k_{r}+1}\right|^{2}\right)
\end{aligned}
$$

for $r=0, \ldots, s-1$ and if $k_{0} \neq 0$. Whereas if $k_{0}=0$,

$$
\Delta \log \left|\operatorname{det} \tilde{A}_{0}\right| \geq m_{1} \kappa+2\left(\left|X_{21}^{0}\right|^{2}-2\left|\tilde{A}_{0}\right|^{2}+\left|A_{1}\right|^{2}\right) \text {. }
$$

Observe that $\left|A_{k_{0}+\cdots+k_{r}}\right|^{2}=\left|\tilde{A}_{k_{0}+\cdots+k_{r}}\right|^{2}$. For $l=0,1, \ldots, s$, set

$$
F_{l}=\sum_{j=k_{-1}+k_{0}+\cdots+k_{l-1}}^{k_{-1}+\cdots+k_{l}-1}\left(k_{-1}+k_{0}+\cdots+k_{l}-j\right) \Delta \log \left|\operatorname{det} A_{j}\right|,
$$

where for notational convenience we set $\operatorname{det} A_{k_{0}+\cdots+k_{r}}=\operatorname{det} \tilde{A}_{k_{0}+\cdots+k_{r}}$, even though the first one does not make sense. We set also $D_{2}^{-1}=0$. Then $F_{l}$, $l=0,1, \ldots, s$, are well-defined continuous functions on $S^{2}$.

A tedious computation shows that

$$
\begin{aligned}
F_{s}+ & \sum_{r=0}^{s-1}\left[\prod_{j=r+1}^{s}\left(k_{j}+1\right)\right] F_{r} \\
\geq & \frac{1}{2} \sum_{r=0}^{s} \prod_{j=r}^{s} k_{r}\left(k_{r}+1\right) m_{r} \kappa+2\left|A_{k_{0}+\cdots+k_{s}}\right|^{2} \\
& -2\left[\prod_{j=1}^{s}\left(k_{j}+1\right)\right]\left(k_{0}+1\right)\left|A_{0}\right|^{2} \\
& +2\left[k_{s}\left|D_{2}^{k_{0}+\cdots+k_{s-1}-1}\right|^{2}+\sum_{r=0}^{s-1} \prod_{j=r+1}^{s}\left(k_{j}+1\right) k_{r}\left|D_{2}^{k_{0}+\cdots+k_{r-1}-1}\right|^{2}\right] \\
\geq & \frac{1}{2} \sum_{r=0}^{s}\left[\prod_{j=r}^{s} k_{r}\left(k_{j}+1\right)\right] m_{r} \kappa-2 \prod_{j=0}^{s}\left(k_{j}+1\right) .
\end{aligned}
$$

Now, if $\kappa \geq k(f)$, then

$$
F_{s}+\sum_{r=0}^{s-1}\left[\prod_{j=r+1}^{s}\left(k_{j}+1\right) F_{r}\right] \geq 0
$$

which means that the function $h$ defined by

$$
h=\prod_{j=0}^{s} h_{j}
$$


is a subharmonic function on $S^{2}$ except for finitely many points, where for $0 \leq l<s$

$$
h_{l}=\log \left[\prod_{j=k_{-1}+\cdots+k_{l-1}}^{k_{-1}+\cdots+k_{l}-1}\left|\operatorname{det} A_{j}\right|^{\left(k_{-1}+\cdots+k_{l}-j\right)}\right]^{\prod_{i=l+1^{\left(k_{i}+1\right)}}^{s}}
$$

and

$$
h_{s}=\log \left[\prod_{j=k_{-1}+\cdots+k_{s-1}}^{k_{-1}+\cdots+k_{s}-1}\left|\operatorname{det} A_{j}\right|^{\left(k_{-1}+\cdots+k_{s}-j\right)}\right] \text {. }
$$

Therefore, $h$ must be constant. It follows from (2.27) and (2.37) that $\kappa=$ $k(f)$.

$$
\begin{gathered}
\left|B_{1}\right|=\left|B_{2}\right|=0, \\
\left|A_{n}\right|=\left|A_{k_{0}+\cdots+k_{s}}\right|=0
\end{gathered}
$$

and

$$
k_{s}\left|D_{2}^{k_{0}+\cdots+k_{s-1}-1}\right|^{2}+\sum_{r=0}^{s-1}\left[\prod_{j=r+1}^{s}\left(k_{j}+1\right) k_{r}\left|D_{2}^{k_{0}+\cdots+k_{r-1}-1}\right|^{2}\right]=0,
$$

which imply that $f$ is holomorphic, $f_{n}$ is antiholomorphic and

$$
k_{s}\left|D_{2}^{k_{0}+\cdots+k_{r-1}-1}\right|^{2}=0,
$$

for $r=1, \ldots, s$. Thus, either $k_{r}=0$ or $D_{2}^{k_{0}+\cdots+k_{r-1}-1}=0$. But when $r \geq 2$, $D_{2}^{k_{0}+\cdots+k_{r-1}-1}=0$ would imply that $\operatorname{rank}\left(\partial f_{k_{0}+\cdots+k_{r-1}-1}\right)=m_{r}<m_{r-1}$, which is a contradiction. Therefore $k_{r}=0$ for $r \geq 2$. Also, $k_{1}=0$, unless $k_{0}=0$. For the latter case by (2.34) and (2.37),

$$
X_{12}=0 .
$$

The pull back of the Maurer-Cartan form is now the following,

$$
\phi=\left[\begin{array}{ccccccc}
\Omega_{11}^{0} & 0 & & & & & \\
0 & \Omega_{22}^{0} & -{ }^{\tau} \bar{A}_{0} \bar{\theta} & & & 0 & \\
& \tilde{A}_{0} \theta & \phi_{1} & -{ }^{\tau} \bar{A}_{1} \bar{\theta} & & & \\
& & A_{1} \theta & \phi_{2} & & & \\
& 0 & & & A_{n-1} \theta & \phi_{n} & 0 \\
& & & & & 0 & \phi_{n+1}
\end{array}\right] .
$$

We see that after a unitary transformation on $C^{N}, f$ can be decomposed as $f=\left[\tilde{f} \oplus V_{0}\right]: S^{2} \rightarrow G(m, N)$, where $V_{0}$ is an $\left(m-m_{1}\right)$-dimensional constant vector subspace of $C^{N}$ and $\tilde{f}$ is a holomorphic map from $S^{2}$ to $G\left(m_{1}, N\right)$, and $\operatorname{rank}\left(f_{j}\right)=m_{1}$, for $j=1, \ldots, n$. In this sense, $f$ essentially generates a Frenet pseudoholomorphic sequences. Q.E.D. 
Remark. Given a harmonic map $f: S^{2} \rightarrow G(m, N)$, one can define an associated vector bundle of $f$ over $S^{2}$ in the following way:

$$
\delta_{f}=\left\{(x, \nu) \in S^{2} \times C^{N} \mid \nu \in f(x)\right\} .
$$

If $f$ generates an orthogonal harmonic sequence then the trivial complex vector bundle

$$
\pi: S^{2} \times C^{N} \rightarrow S^{2}
$$

splits into orthogonal complex vector subbundles,

$$
S^{2} \times C^{N}=\delta_{f} \oplus \delta_{f_{1}} \oplus \cdots \oplus \delta_{f_{n}} \oplus \delta,
$$

which are, in fact, holomorphic subbundles. Here $\delta$ is the orthogonal complement of $\delta_{f} \oplus \cdots \oplus \delta_{f_{n}}$. Theorem 2.3 says that if the curvature is large, then the ranks of $\delta_{f_{j}}, j=1, \ldots, n$, are equal, $f$ is holomorphic and $\delta$ is a trivial subbundle.

We say that a map $g$ is in a Frenet harmonic sequence (i.e. constant ranks and orthogonal)

$$
f \equiv f_{0} \stackrel{\partial}{\rightarrow} f_{1} \stackrel{\partial}{\rightarrow} f_{2} \rightarrow \cdots \rightarrow f_{n}
$$

of positive $j$, if $g=f_{j}$ for some $j=0, \ldots, n$, where $f$ is harmonic and $\operatorname{rank}\left(f_{j}\right)=m$. For such a map, we show the following pinching theorem.

Theorem 2.4. Let $g=f_{j}: S^{2} \rightarrow G(m, N)$ be a harmonic isometric immersion, which is in the above Frenet harmonic sequence, and let $\kappa_{j}$ be the Gaussian curvature. Suppose that $\kappa_{j} \geq 4 / m[2 j(n-j)+n]$, then $\kappa_{j}=4 / m[2 j(n-j)+n]$ is constant, and the directrix $f$ is holomorphic.

Proof. We use the invariants developed in the proof of Theorem 2.3. These invariants now satisfy

$$
\begin{gathered}
\Delta \log \left|\operatorname{det} A_{0}\right|=m \kappa_{j}+2\left(\left|A_{1}\right|^{2}-2\left|A_{0}\right|^{2}+\left|B_{1}\right|^{2}+\left|B_{2}\right|^{2}\right), \\
\Delta \log \left|\operatorname{det} A_{\tau}\right|=m \kappa_{j}+2\left(\left|A_{\tau-1}\right|^{2}+\left|A_{\tau+1}\right|^{2}-2\left|A_{\tau}\right|^{2}\right)
\end{gathered}
$$

for $0<\tau<n-1$, and

$$
\Delta \log \left|\operatorname{det} A_{n-1}\right|=m \kappa_{j}+2\left(\left|A_{n-2}\right|^{2}+\left|A_{n}\right|^{2}-2\left|A_{n-1}\right|^{2}+\left|B_{1}\right|^{2}\right) .
$$

$\left(1.11^{\prime}\right)$ becomes

$$
\begin{gathered}
\left|A_{j-1}\right|^{2}+\left|A_{j}\right|^{2}=1, \quad \text { if } j \neq 0, n-1, \\
\left|A_{0}\right|^{2}+\left|B_{1}\right|^{2}+\left|B_{2}\right|^{2}=1, \quad \text { if } j=0,
\end{gathered}
$$

and

$$
\left|A_{n-1}\right|^{2}+\left|A_{n}\right|^{2}+\left|B_{1}\right|^{2}=1, \quad \text { if } j=n-1 .
$$


The case when $j=0$ has been treated in Theorem 2.3 by setting $m_{r}=m$ for $0 \leq r \leq s, k_{0}=n$ and $k_{1}=k_{2}=\cdots=0$. When $j>0$, set

$$
h_{j}=\left[\prod_{i=0}^{j-1}\left|\operatorname{det} A_{i}\right|^{(2 j+1)(n-i-1)+2 i}\right] \cdot\left[\prod_{i=j}^{n-1}\left|\operatorname{det} A_{i}\right|^{(2 j+1)(n-i)}\right] \text {. }
$$

Then

$$
\begin{aligned}
\Delta \log h_{j}= & \frac{n+1}{2}\left[(2 j(n-j)+n) m \kappa_{j}-4\left(\left|A_{j}\right|^{2}+\left|A_{j-1}\right|^{2}\right)\right] \\
& +2(2 j+1)(n-1)\left(\left|B_{1}\right|^{2}+\left|B_{2}\right|^{2}\right)+2(2 j+1)\left(\left|A_{n}\right|^{2}+\left|B_{1}\right|^{2}\right) \\
\geq & \frac{n+1}{2}\left[(2 j(n-j)+n) m \kappa_{j}-4\right]
\end{aligned}
$$

The assumption on $\kappa_{j}$ implies

$$
\Delta \log h_{j} \geq 0
$$

which means that $h_{j}$ is constant, $\kappa_{j}=\frac{4}{m[2 j(n-j)+n]}$, and $A_{n}=B_{1}=B_{2}=0$. Therefore the directrix $f$ is holomorphic. Q.E.D.

We say that a map $f$ from $M$ into $C P^{n}$ is linearly full, if $f(M)$ is not contained in some linear subspace $C P^{n^{\prime}} \subset C P^{n}$, where $n^{\prime}<n$. The following corollary was first proved in [BJRW].

Corollary 2.4. For a linearly full pseudoholomorphic map $f_{j}$ of positions $j$ from $S^{2}$ into $C P^{n}$, if it is an isometric immersion, and if the curvature $\kappa_{j} \geq$ $4 /(2 j(n-j)+n)$, then $\kappa_{j}=4 /(2 j(n-j)+n)$ is constant.

\section{HARMONIC TWO-SPHERES IN $G(2,4)$ AND $G(2,6)$}

In this section, we look at harmonic maps from $S^{2}$ into $G(2,4)$ and $G(2,6)$.

Let $f$ be a harmonic isometric immersion from $S^{2}$ into $G(2,4)$. By Corollary 2.2 , we know that at least one of the $\partial$-transform and the $\bar{\partial}$-transform is degenerate. Let us say rank $(\partial f) \leq 1$. Choosing a suitable local unitary frame $e=\left(e_{1}, e_{2}, e_{3}, e_{4}\right)$ along $f$ as before, the pull back of the Maurer-Cartan form is

where

$$
\phi=\left[\begin{array}{cccc}
\phi_{11} & \phi_{12} & -\bar{a}_{31} \bar{\theta}-\bar{b}_{31} \theta & -\bar{b}_{41} \theta \\
\phi_{21} & \phi_{22} & -\bar{b}_{32} \theta & -\bar{b}_{42} \theta \\
a_{31} \theta+b_{31} \bar{\theta} & b_{32} \bar{\theta} & \phi_{33} & \phi_{34} \\
b_{41} \bar{\theta} & b_{42} \bar{\theta} & \phi_{43} & \phi_{44}
\end{array}\right],
$$

$$
\left|a_{31}\right|^{2}+\left|b_{31}\right|^{2}+\left|b_{32}\right|^{2}+\left|b_{41}\right|^{2}+\left|b_{42}\right|^{2}=1 .
$$

From Corollary 2.1, the vanishing of $\operatorname{tr} C=\operatorname{tr}^{t} A \bar{B}$ implies

$$
a_{31} \bar{b}_{31}=0 \text {. }
$$


Harmonicity conditions $\left(1.12^{\prime}\right)$ and $\left(1.13^{\prime}\right)$ imply

$$
\begin{gathered}
d a_{31}=a_{31}\left(-i w+\phi_{11}-\phi_{33}\right) \quad \bmod \theta, \\
\phi_{12}=a_{12} \theta
\end{gathered}
$$

and

$$
\phi_{43}=a_{43} \theta \text {. }
$$

By Proposition 1.1, (3.3) means that $a_{31}$ is a function of analytic type. Thus either it is identically zero which means that $f$ is an antiholomorphic map or away from its zeros it satisfies

$$
\Delta \log \left|a_{31}\right| \theta \wedge \bar{\theta}=2 d\left(-i w+\phi_{11}-\phi_{33}\right) .
$$

Suppose that $f$ is not antiholomorphic. Then $b_{31}=0$. Harmonicity condition (1.13) also implies

$$
\begin{gathered}
d b_{32}=b_{32}\left(i w+\phi_{22}-\phi_{33}\right) \quad \bmod \bar{\theta}, \\
d b_{41}=b_{41}\left(i w+\phi_{11}-\phi_{44}\right)
\end{gathered}
$$

and

$$
d b_{42}=b_{42}\left(i w+\phi_{22}-\phi_{44}\right)+b_{41} \phi_{12}-b_{32} \phi_{43} \bmod \bar{\theta}
$$

from which it follows again that $b_{32}$ and $b_{41}$ are functions of analytic type.

If $b_{32}$ is not identically zero, then away from its zeros we get

$$
\Delta \log \left|b_{32}\right| \theta \wedge \bar{\theta}=2 d\left(-i w+\phi_{33}-\phi_{22}\right) .
$$

Combining (3.6) and (3.10) and using the Maurer-Cartan structure equations, we have

$$
\begin{aligned}
\Delta \log \left|a_{31} b_{32}\right|= & 2 \kappa+4\left|a_{12}\right|^{2}-2\left|a_{31}\right|^{2}+2\left|b_{41}\right|^{2} \\
& -2\left|b_{32}\right|^{2}-2\left|b_{42}\right|^{2} \\
\geq & 2 \kappa-2 .
\end{aligned}
$$

Notice that the function $\left|a_{31} b_{32}\right|$ is a globally defined continous function. If $\kappa \geq 1$, then we see that $\kappa=1$ and $a_{12}=b_{41}=0$. Notice also that the local unitary frame $e$ is uniquely determined up to $U(1) \times U(1) \times U(1) \times$ $U(1)$-transformations, and thus for $i=1,2,3$ and 4 , each $e_{i}$ defines a map $\left[e_{i}\right]: S^{2} \rightarrow C P^{3}$. In this case since $a_{12}=b_{41}=b_{31}=0,\left[e_{1}\right]$ is holomorphic, $\left[e_{2}\right]$ is antiholomorphic and $f=\left[e_{1} \oplus e_{2}\right]$.

The other case is $b_{32}=0$. Suppose $b_{41}$ is not identically zero. Then by (3.8) and (3.6), we get

$$
\begin{aligned}
\Delta \log \left|b_{41} a_{31}\right| & =2 \kappa+4\left|a_{43}\right|^{2}-2\left|b_{42}\right|^{2}-2\left|b_{41}\right|^{2}-2\left|a_{31}\right|^{2} \\
& \geq 2 \kappa-2 .
\end{aligned}
$$

Again, if $\kappa \geq 1$, then $\kappa=1$ and $a_{43}=0$. In this case, we see that $\left[e_{3}\right]$ is an antiholomorphic map, $\left[e_{4}\right]$ is a holomorphic map and $f=\left[e_{3} \oplus e_{4}\right]^{\perp}$. 
Finally, if $b_{32}=b_{41}=0$, but $b_{42}$ is not identically zero, then by (3.9),

$$
\Delta \log \left|b_{42}\right| \theta \wedge \bar{\theta}=2 d\left(-i w+\phi_{44}-\phi_{22}\right) .
$$

Taking the exterior derivatives on both sides of (3.4) and (3.5), and using the structure equations, we get

$$
d a_{12}=a_{12}\left(-i w-\phi_{11}+\phi_{22}\right) \quad \bmod \theta
$$

and

$$
d a_{43}=a_{43}\left(-i w+\phi_{33}-\phi_{44}\right) \quad \bmod \theta .
$$

Repeating the previous argument, we see that both $a_{12}$ and $a_{43}$ are of analytic type and that $\left|a_{12}\right|$ and $\left|a_{43}\right|$ are globally defined on $S^{2}$.

We claim that if $k \geq 1$, then $a_{12}$ and $a_{43}$ are identically zero. There are three cases to be checked.

Case (i). Both $a_{12}$ and $a_{43}$ are not identically zero. Then from (3.14) and (3.15),

$$
\Delta \log \left|a_{12}\right| \theta \wedge \bar{\theta}=2 d\left(-i w-\phi_{11}+\phi_{22}\right)
$$

and

$$
\Delta \log \left|a_{43}\right| \theta \wedge \bar{\theta}=2 d\left(-i w+\phi_{33}-\phi_{44}\right) .
$$

These, together with (3.6) and (3.13), would give

$$
\Delta \log \left|a_{12} a_{31} a_{43} b_{42}\right|=4 k \geq 4>0 .
$$

But then $\left|a_{12} a_{31} a_{43} b_{42}\right|$ must be constant and $k=0$, which is a contradiction. Case (ii). $a_{12}=0$, but $a_{43}$ is not zero. Then (3.17) holds and the combination of (3.6), (3.13) and (3.17) gives

$$
\Delta \log \left|a_{31} a_{43} b_{42}\right|=3 k-2>1,
$$

which again would imply $k=\frac{2}{3}$, contradicting the hypothesis on $k$.

Case (iii). $a_{43}=0$, but $a_{12}$ is not zero. This case can be treated in exactly the same way as Case (ii), and it cannot happen either.

Now since $a_{12}=a_{43}=0$, by reading the pull back of the Maurer-Cartan form we see that $e_{1}$ defines a holomorphic map $\left[e_{1}\right]: S^{2} \rightarrow C P^{1} \subset C P^{3}$, and that $e_{2}$ defines an antiholomorphic map $\left[e_{2}\right]: S^{2} \rightarrow C P^{1} \subset C P^{3}$. Up to a unitary transformation the map $f$ is then given by $f=h \circ g$ in the following way.

$$
\frac{f}{\widehat{S^{2} \rightarrow C P^{1} \times C P^{1} \underset{h}{\rightarrow} C} P^{3}}
$$


where $g=\left[e_{1}\right] \times\left[e_{2}\right]: S^{2} \rightarrow C P^{1} \times C P^{1}$ and $h$ is the holomorphic embedding given by

$$
\begin{aligned}
& h\left(\left[Z_{0}, Z_{1}\right],\left[Z_{2}, Z_{3}\right]\right) \\
& \quad=\left[\frac{Z_{0}}{\sqrt{\left|Z_{0}\right|^{2}+\left|Z_{1}\right|^{2}}}, \frac{Z_{1}}{\sqrt{\left|Z_{0}\right|^{2}+\left|Z_{1}\right|^{2}}}, \frac{Z_{2}}{\sqrt{\left|Z_{2}\right|^{2}+\left|Z_{3}\right|^{2}}}, \frac{Z_{3}}{\sqrt{\left|Z_{2}\right|^{2}+\left|Z_{3}\right|^{2}}}\right] .
\end{aligned}
$$

In summary, we have shown

Theorem 3.1. Let $f$ be a harmonic isometric immersion from $S^{2}$ into $G(2,4)$ with $\operatorname{rank}(\partial f)=1$ and $\operatorname{rank}(\bar{\partial} f) \geq 1$. If the curvature $\kappa \geq 1$, then either $f=$ $\left[e_{1} \oplus e_{2}\right]$ or $f^{\perp}=\left[e_{1} \oplus e_{2}\right]$ with $\left[e_{1}\right]: S^{2} \rightarrow C P^{3}$ holomorphic, $\left[e_{2}\right]: S^{2} \rightarrow C P^{3}$ antiholomorphic and $e_{1} \perp e_{2}$. Moreover, if the curvature $\kappa$ is not constant 1 , then up a unitary transformation $f$ is given by (3.20).

Remark. Suppose now that $\kappa \geq 2$. Then it happens only when $a_{12}=a_{43}=$ $b_{31}=b_{32}=b_{41}=0$. From (3.6) and (3.13), we set that

$$
\Delta \log \left|a_{31} b_{42}\right|=2 k-4 \geq 0 .
$$

Thus $k=2$ and $\left|a_{31} b_{42}\right|$ is constant. But by (3.1),

$$
\left|a_{31}\right|^{2}+\left|b_{42}\right|^{2}=1,
$$

and thus both $\left|a_{31}\right|$ and $\left|b_{31}\right|$ are constant. We can specify the unitary frame $e$ so that $a_{31} \geq 0$ and $b_{31} \geq 0$ are constant. Using (3.6), it is easy to see that $a_{31}=b_{42}=\frac{1}{2}$. Thus all invariants are constant. In this case up to a $U(4)$-congruence, $f$ is the map $V_{1, \overline{1}}$ defined by

$$
V_{1, \overline{1}}\left[\begin{array}{l}
Z_{0} \\
Z_{1}
\end{array}\right]=\left[\begin{array}{cc}
Z_{0} & 0 \\
Z_{1} & 0 \\
0 & \bar{Z}_{0} \\
0 & \bar{Z}_{1}
\end{array}\right] .
$$

Notice that the map $V_{1,0}: S^{2} \rightarrow G(2,4)$, defined by

$$
V_{1,0}\left[\begin{array}{l}
Z_{0} \\
Z_{1}
\end{array}\right]=\left[\begin{array}{cc}
Z_{0} & 0 \\
Z_{1} & 0 \\
0 & 1 \\
0 & 0
\end{array}\right],
$$

is the only holomorphic map from $S^{2}$ to $G(2,4)$ with constant curvature 4 up to $U(4)$-congruences. In [CZ], we also classified all holomorphic maps from $S^{2}$ to $G(2,4)$ of curvature 2 . In fact, the argument in [CZ] actually shows that for a holomorphic isometric immersion $f$ from $S^{2}$ to $G(2,4)$, if the curvature $\kappa \geq 2$, then either $\kappa=2$ or $\kappa=4$. Theorem 3.1, together with the argument in $[\mathrm{CZ}]$, shows the following. 
Theorem 3.2. Up to U(4)-congruence, all isometric harmonic maps from $S^{2}$ into $G(2,4)$ of curvature $\kappa \geq 2$ are given by: $V_{1, \overline{1}}, V_{1,0}$, and the holomorphic curves (and their complex conjugates) of curvature 2 given in [CZ].

Now suppose that $f$ is a harmonic isometric immersion from $S^{2}$ into $G(2,6)$. Then the only nonholomorphic or nonantiholomorphic cases are those when $\operatorname{rank}(\partial f) \geq 1$ and $\operatorname{rank}(\bar{\partial} f) \geq 1$. These cases can be treated by using Theorem 2.2. In fact, we have the following theorem.

Theorem 3.3. For a harmonic isometric immersion $f$ from $S^{2}$ into $G(2,6)$, IF $\operatorname{rank}(\partial f)=\operatorname{rank}(\bar{\partial} f)=2$, and if the curvature $\kappa \geq \frac{1}{2}$, then $\kappa=\frac{1}{2}$ is constant and $f$ is a pseudoholomorphic curve of position one; if $\operatorname{rank}(\bar{\partial} f)=2$ and $\operatorname{rank}(\bar{\partial} f)=1$, or if $\operatorname{rank}(\partial f)=1$ and $\operatorname{rank}(\bar{\partial} f)=2$, then $\min \kappa<\frac{8}{7}$; finally, if $\operatorname{rank}(\partial f)=\operatorname{rank}(\bar{\partial} f)=1$, and if $\kappa \geq 2$, then $\kappa=2$ is constant.

\section{REFERENCES}

[BJRW] J. Bolton, G. R. Jense, M. Rigoli, and L. M. Woodward, On conformal minimal immersions of $S^{2}$ into $C P^{n}$, Math. Ann. 279 (1988), 599-620.

[BRS] F. Burstall, J. Rawnsley and S. Salamon, Stable harmonic 2-spheres in symmetric spaces, Bull. Amer. Math. Soc. 16 (1987), 274-278.

[CW] S. S. Chern and J. G. Wolfson, Harmonic maps of the two-sphere into a complex Grassmann manifold. II, Ann. of Math. 125 (1987), 301-335.

[CZ] Q. Chi and Y. Zheng, Rigidity of pseudo-holomorphic curves of constant curvature in Grassmann manifolds, Trans. Amer. Math. Soc. 313 (1989), 393-406.

[DZ] A. M. Din and W. J. Zakrzewski, General classical solutions in the $C P^{n-1}$ model, Nuclear Phys. B 174 (1980), 397-406.

[EW] J. Elles and J. C. Wood, Harmonic maps from surfaces to complex projective spaces, Adv. in Math. 49 (1983), 217-263.

[EGT] J. H. Eschenburg, I. V. Guadalupe and R. Tribuzy, The fundamental equation of minimal surfaces in $C P^{2}$, Math. Ann. 270 (1985), 571-598.

[Ra] J. Ramanathan, Harmonic maps from $S^{2}$ to $G(2,4)$, J. Differential Geometry 19 (1984), 207-219.

[R] M. Rigoli, A rigidity result for holomorphic emmersions of surfaces in $C P^{n}$, Proc. Amer. Math. Soc. 93 (1985), 317-320.

[Z] Y. Zheng, Harmonic maps into Grassmann manifolds, Washington Univ. Thesis, 1987.

Department of Mathematics, Rice University, Houston, Texas 77251

Current address: 2608 Snead Drive, Alhambra, California 91803 